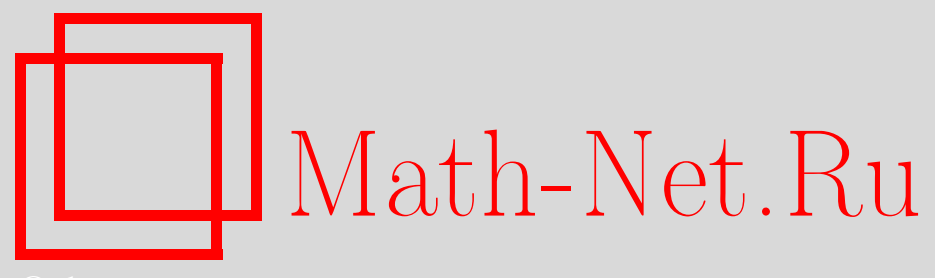

А. В. Шаповалов, Характеристики случайных систем дискретных уравнений при неравновероятной выборке неизвестных, Матем. вопр. криптогр., 2010, том 1, выпуск 3, 93-117

DOI: https://doi.org/10.4213/mvk17

Использование Общероссийского математического портала Math-Net.Ru подразумевает, что вы прочитали и согласны с пользовательским соглашением http://www.mathnet.ru/rus/agreement

Параметры загрузки:

IP : 54.172 .240 .79

26 апреля 2023 г., 14:39:09 
УДК 519.212.2

\title{
Характеристики случайных систем дискретных уравнений при неравновероятной выборке неизвестных
}

\author{
Шаповалов А. В.
}

Лаборатория ТВП, Москва

Получено 22.IV.2010

Изучается случайная система дискретных уравнений $S$ относительно $n$ неизвестных, состоящая из $M=M(n)$ уравнений. Каждое уравнение содержит не более $m$ неизвестных, которые выбираются случайно, независимо и, вообще говоря, неравновероятно. Указаны условия, при которых предельное при $M-c \sqrt{n}=o(\sqrt{n})$, $n \rightarrow \infty, m=$ const значение вероятности совместности случайной системы уравнений непрерывно убывает от 1 до 0 с ростом $c$ от 0 до $\infty$. Построен алгоритм распознавания несовместности случайной системы уравнений, имеющий трудоемкость $O(\sqrt{n})$. Предел вероятности распознавания несовместности для этого алгоритма такой же, как для алгоритма полного перебора. Доказательства используют геометрические свойства случайной системы уравнений.

Ключевые слова: системы дискретных уравнений, неразрешимость, вероятностные алгоритмы

\section{Properties of random systems of discrete equations with nonuniform sampling of unknowns}

\section{A. V. Shapovalov \\ TVP Laboratory, Moscow}

Abstract. A random system $S$ of $M=M(n)$ discrete equations with $n$ unkowns is considered. Each equation contains at most $m$ unknowns which are selected at random, independently and (possibly) nonuniformly. We find conditions on the structure of equations ensuring that for $M-c \sqrt{n}=o(\sqrt{n}), n \rightarrow \infty, m=$ const, the limit probability of solvability decreases continuously from 1 to 0 when $c$ increases from 0 to $\infty$. An algorithm detecting the unsolvability of a random system of equations is described. This algorithm has low time complexity $O(\sqrt{n})$. The limiting probability of detection the unsolvability is the same as for the exhaustive search algorithm. Our proofs are based on the geometric properties of random system of equations.

Key words: systems of discrete equations, unsolvability, probabilistic algorithms

Citation: Mathematical Aspects of Cryptography, 2010, vol. 1, no. 3, pp. 93-117 (Russian). 


\section{1. Введение}

Пусть $\Phi_{i}=\left\{f_{i, 1}, f_{i, 2}, \ldots, f_{i, \mid \Phi_{i} l}\right\}, i=1, \ldots, m$, - упорядоченные множества конечных дискретных функций, заданных на множестве $i$-мерных векторов, координаты которых выбираются из конечного множества $B=\left\{b_{1}, \ldots, b_{|B|}\right\}$, $|B| \geq 2$. Каждая функция $f_{i, j}\left(y_{1}, \ldots, y_{i}\right)$ из $\Phi_{i}$ существенно зависит ровно от $i$ переменных и принимает значения в конечном множестве $A=\left\{a_{0}, \ldots, a_{z-1}\right\}$, $z>1$. Через

$$
\mathbf{E}=\{1, \ldots, m\} \times\left\{1,2, \ldots, \max _{i=0,1, \ldots, m}\left|\Phi_{i}\right|\right\} \times A
$$

обозначим декартово произведение трех указанных множеств. Пусть $\left\{c_{i, j, a},(i, j, a) \in E\right\}$ - набор неотрицательных констант, сумма которых равна единице; если $j>\left|\Phi_{i}\right|$ или $a$ не принадлежит множеству значений функции $f_{i, j}$, то $c_{i, j, a}=0$. Для каждого $n, n=2,3, \ldots$, определены вероятности $p_{k}=p_{k}(n), k=1, \ldots, n$, удовлетворяющие соотношениям

$$
\begin{gathered}
p_{1}+\ldots+p_{n}=1, \varepsilon_{1}<n p_{k}<\varepsilon_{2}, k=1, \ldots, n, \\
\lim _{n \rightarrow \infty}\left(n \sum_{k=1}^{n} p_{k}^{2}\right)=\sigma^{2},
\end{gathered}
$$

где $\varepsilon_{1}, \varepsilon_{2}, \sigma^{2}$ - некоторые положительные константы.

Случайная система уравнений $S=S_{n, M, \bar{c}}$ относительно неизвестных $x_{1}, \ldots, x_{n}$ состоит из $M$ упорядоченных уравнений, неизвестные могут принимать значения в множестве $B$. Уравнения в случайной системе уравнений $S$ не зависят друг от друга, выбираются последовательно, вероятность появления уравнения вида

$$
f_{i, j}\left(x_{s_{1}}, \ldots, x_{s_{i}}\right)=a
$$

равна $c_{i, j, a}$ для каждого набора $(i, j, a) \in E$. Каждый индекс $s_{1}, \ldots, s_{i}$ принимает значения $1, \ldots, n$ случайно и независимо от других индексов с вероятностями $p_{1}, \ldots, p_{n}$ соответственно (выборка с возвращением).

Индексы $i$ и $j$ в (3) означают, что функция $f_{i, j}$ в левой части уравнения является $j$-м элементом множества $\Phi_{i}$. Вероятность появления в левой части уравнения случайной системы уравнений $S$ функции $f_{i, j}$ равна $\sum_{a \in A} c_{i, j, a}=c_{i, j}$.

Тогда при $c_{i, j}>0$ величина $c_{i, j, a} / c_{i, j}$ есть вероятность появления $a \in A$ в правой части уравнения случайной системы уравнений $S$ при условии, что в левой части этого уравнения находится функция $f_{i, j}$. Поэтому в случайной системе уравнений $S$ значения правых частей уравнений, вообще говоря, 
зависят от вида функций $f_{i, j}$ в их левых частях. Случайная система уравнений $S$ является случайной системой уравнений с независимыми левыми и правыми частями (см., например, $[1-4,7,8,13])$ тогда и только тогда, когда для каждого $a \in A$ и всех пар $(i, j)$, таких, что $c_{i, j}>0$, вероятности $c_{i, j, a} / c_{i, j}$ не зависят от $i$ и $j: c_{i, j, a} / c_{i, j}=p(a)$.

В работе исследуется асимптотическое поведение вероятности совместности случайной системы уравнений $S$ при $n \rightarrow \infty$, когда число ее уравнений $M=M(n)$ зависит от $n$. Все асимптотические формулы приводятся при $n \rightarrow \infty$.

Под вероятностью некоторого свойства случайной системы уравнений (случайного гиперграфа) понимается сумма вероятностей реализаций соответствующего случайного элемента, обладающих этим свойством. Вероятность совместности $\mathbf{P}_{c}(S)$ случайной системы уравнений $S$ равна сумме вероятностей совместных реализаций этой случайной системы уравнений. При заданном соотношении параметров $n$ и $M(n)$ почти все реализации случайной системы уравнений или случайного гиперграфа обладают (не обладают) свойством $Q$, если вероятность этого свойства $\mathbf{P}(Q)$ стремится к единице (к нулю) при $n \rightarrow \infty$. Если существует функция $Q(n)$, монотонно возрастающая при $n \rightarrow \infty$ и такая, что $\mathbf{P}_{c}(S)$ стремится к единице (нулю) при $M(n) / Q(n) \rightarrow 0(M(n) / Q(n) \rightarrow \infty)$, то она называется пороговой функиией совместности случайной системы уравнений $S$.

В работе используются обозначения $(x)_{k}=x(x-1) \ldots(x-k+1) ; \mathbf{E}(\zeta)_{r}=$ $=\mathbf{E} \zeta(\zeta-1) \ldots(\zeta-r+1)-r$-й факториальный момент случайной величины $\zeta$; запись множества $\left\{x_{1}, \ldots, x_{s}\right\}$ в виде $\left\langle x_{1}, \ldots, x_{s}\right\rangle$ означает, что его элементы упорядочены; $|X|$ — число элементов в конечном множестве $X ; X \backslash Y$ разность множеств $X$ и $Y ; \varnothing-$ пустое множество.

\section{2. Основные результаты}

Будем говорить, что элемент $b \in B$ является допустимым (недопустимым, или запретным) значением переменной $x_{s_{k}}$ в уравнении (3), если при разных индексах $s_{1}, \ldots, s_{i}$ это уравнение имеет (не имеет) решение, в котором $x_{s_{k}}=b$, где $x_{s_{k}} \in\left\{x_{s_{1}}, \ldots, x_{s_{i}}\right\}$. Под множеством допустимых (недопустимых, или запретных) значений переменной $x_{s_{k}}$ в уравнении (3) будем понимать множество всех допустимых (запретных) значений переменной $x_{s_{k}}$ в уравнении (3). Переменная $x_{s_{k}}$ частично определяется из уравнения (3), если множество ее допустимых значений является собственным подмножеством множества $B$. 
Упорядочим некоторым образом множество всех подмножеств множества $B:\left\{B_{0}, B_{1}, \ldots, B_{h}\right\}$, где $h=2^{|B|}-1$, пусть $B_{0}=\varnothing, B_{h}=B$. Для каждого $B_{s}$, $s=0,1, \ldots, h$, обозначим через $t_{i, j, a}\left(B_{s}\right)$ число переменных уравнения (3), имеющих множество допустимых значений $B_{s}$, и

$$
\chi_{s}=\sum_{(i, j, a) \in E} c_{i, j, a} t_{i, j, a}\left(B_{s}\right) .
$$

Вероятностный смысл величины $\chi_{s}$ - среднее значение числа переменных, имеющих множество допустимых значений $B_{s}$ в одном уравнении, случайно выбираемом для реализации случайной системы уравнений $S$ в соответствии с ее вероятностной мерой, $\chi_{0} \equiv 0$.

Теорема 1. Случайная система уравнений $S$ имеет пороговую функцию совместности $\sqrt{n}$ тогда и только тогда, когда $\chi=\sum_{B_{s} \cap B_{k}=\varnothing} \chi_{s} \chi_{k}>0$. При условии

$$
M \sim c \sqrt{n},
$$

где с >0 - константа, справедлива формула

$$
\mathbf{P}_{c}(S)=e^{-c^{2} \sigma^{2} \chi}+o(1) .
$$

Теорема 2. Почти все реализачии случайной системы уравнений $S$ совместны при $M=o\left(n^{2 / 3}\right)$ тогда и только тогда, когда $\chi=0$.

Если $\chi_{s}=0$ для каждого $B_{s}, s=1, \ldots, h-1$, то $\chi=0$.

Следующий алгоритм эффективно решает задачу распознавания несовместности реализаций случайной системы уравнений $S$ при $\chi>0$. Цель алгоритма - найти два уравнения с несовместными множествами допустимых значений для одного неизвестного: $x_{v} \in B_{s}, x_{v} \in B_{k}, B_{s} \cap B_{k}=\varnothing$, из которых следует несовместность системы уравнений.

\section{Алгоритм $A$}

1. Предварительно для каждого набора $(i, j, a) \in E$ при $c_{i, j, a}>0$ проводится классификация частично определяющихся из уравнения (3) переменных, включая множества их запретных значений, и создаётся заполненная нулями матрица $L$ размера $n \times|B|$. Значение 1 элемента на пересечении $v$-й строки и $t$-го столбца матрицы будет означать, что $x_{v} \neq b_{t}$, а значение 0 этого элемента - отсутствие такого ограничения, $b_{t} \in B, v=1, \ldots, n, t=1, \ldots,|B|$.

2. Последовательно перебираются уравнения системы уравнений, которые имеют переменные, частично определяющиеся из этих уравнений. При появлении уравнения, в котором переменная $x_{v}$ имеет множество за- 
претных значений $\left\{b_{t_{1}}, \ldots, b_{t_{r}}\right\}$, в $v$-ю строку на места с номерами $t_{1}, \ldots, t_{r}$ заносятся единицы. Если после этого все элементы $v$-й строки равны 1 , делается вывод о несовместности системы. Если перебраны все уравнения и не появилась строка из одних единиц, работа алгоритма завершается без какого-либо заключения о совместности системы.

Под вероятностью распознавания несовместности (случайно выбираемой) реализации случайной системы уравнений $S$ алгоритмом $\boldsymbol{A}$ понимается сумма вероятностей реализации случайной системы уравнений $S$, несовместность которых этот алгоритм распознает. Максимально возможное значение этой величины - вероятность несовместности случайной системы уравнений $S$, равная $1-\mathbf{P}_{c}(S)$. Надежностью $\pi_{n}$ алгоритма $\boldsymbol{A}$ при $M>1$ будем называть вероятность распознавания несовместности (случайно выбираемой) реализации случайной системы уравнений $S$ алгоритмом $\boldsymbol{A}$ при условии несовместности этой реализации. Отсюда следует, что $0 \leq \pi_{n} \leq 1$. Алгоритм $\boldsymbol{A}$ не совершает ошибок: он может распознать несовместность несовместной реализации (хотя и не каждой), но не может признать несовместной совместную реализацию случайной системы уравнений. Поэтому при $M>1$ надежность алгоритма $\boldsymbol{A}$ равна отношению суммы вероятностей реализаций случайной системы уравнений $S$, несовместность которых алгоритм $\boldsymbol{A}$ распознает, к вероятности несовместности случайной системы уравнений.

Под минимальной несовместной подсистемой системы уравнений будем понимать несовместную подсистему, которая становится совместной при исключении из нее любого уравнения. Почти все несовместные реализации случайной системы уравнений обладают свойством $Q$, если вероятность свойства $Q$ при условии ее несовместности стремится к 1 при $n \rightarrow \infty$.

Теорема 3. Пусть $\chi>0$ и выполнено условие (4) либо $M / \sqrt{n} \rightarrow \infty$. Тогда надежность алгоритма А удовлетворяет формуле $\pi_{n} \sim 1$ и почти все несовместныле реализачии случайной системы уравнений $S$ содержат несовместные подсистемь из двух уравнений, пересекающихся только по одной частично определяющейся из этих уравнений переменной, которая в одном уравнении имеет множество допустимых значений $B_{s}$, a в другом - $B_{k}$ причем $B_{s} \cap B_{k}=\varnothing$. При дополнительном условии $M=o\left(n^{2 / 3}\right)$ почти все несовместные реализачии случайной системы уравнений $S$ не имеют других несовместных подсистем.

В соответствии с теоремами 1 и 3 при $M / \sqrt{n} \rightarrow \infty, \chi>0$ почти все реализации случайной системы уравнений $S$ несовместны, поэтому алгоритм $\boldsymbol{A}$ распознает несовместность почти всех ее реализаций.

Для алгоритма $\boldsymbol{U}$ полного перебора решений случайной системы уравнений $S$ аналогично определяются надежность и вероятность распознавания 
несовместности реализации случайной системы уравнений $S$. Этот алгоритм имеет максимально возможные надежность и вероятность распознавания несовместности реализации случайной системы уравнений $S$, которые тождественно равны 1 и $1-\mathbf{P}_{c}(S)$ соответственно. В условиях теоремы 3 алгоритм $\boldsymbol{A}$ имеет такие же предельные надежность и вероятность распознавания несовместности реализации случайной системы уравнений $S$, как и у алгоритма полного перебора решений.

Сравним трудоемкость алгоритмов $\boldsymbol{A}$ и $\boldsymbol{U}$ при условии (4). Определим среднюю трудоемкость $T_{\mathrm{cp}}(\boldsymbol{A})$ и $T_{\mathrm{cp}}(\boldsymbol{U})$ алгоритмов $\boldsymbol{A}$ и $\boldsymbol{U}$ как среднее значение случайной величины, равной трудоемкости решения задачи распознавания несовместности случайно выбираемой реализации случайной системы уравнений $S$. Пусть максимальная трудоемкость $T_{\max }(\boldsymbol{A})$ и $T_{\max }(\boldsymbol{U})$ алгоритмов $\boldsymbol{A}$ и $\boldsymbol{U}$ равна максимальному значению трудоемкости решения задачи распознавания несовместности реализации случайной системы уравнений $S$. Пусть алгоритм $\boldsymbol{U}$ тратит не менее $k_{1} \geq 1$ операций на проверку совместности одного уравнения при подстановке одного решения. Вероятность случайного выбора совместной реализации случайной системы уравнений $S$ равна $\mathbf{P}_{c}(S)$. Следовательно, при $\lim _{n \rightarrow \infty} \mathbf{P}_{c}(S) \geq \rho>0$ справедлива оценка

$$
T_{\mathrm{cp}}(\boldsymbol{U}) \geq \mathbf{P}_{c}(S) k_{1} M|B|^{n} .
$$

Так как случайная система уравнений $S$ при любом числе уравнений имеет совместную реализацию, то $T_{\max }(\boldsymbol{U})=k_{1} M|B|^{n}$.

В алгоритме $\boldsymbol{A}$ классификация частично определяющихся переменных и множеств запретных значений этих переменных из уравнения вида (3) для конечного числа таких уравнений требует не более некоторого ограниченного числа $K$ операций. Пусть для занесения в матрицу $L$ информации о запретных значениях одной переменной и проверке равенства единице всех элементов одной строки матрицы требуется не более $k_{2}$ операций. Число уравнений в каждой системе равно $M$, число переменных в каждом уравнении не более $m$, поэтому $T_{\max }(\boldsymbol{A})=O\left(K+m k_{2} M\right)=O(M)$ и $T_{\mathrm{cp}}(\boldsymbol{A})=O(M)$.

Таким образом, при условии (4) и $\chi>0$ алгоритм $\boldsymbol{A}$ имеет максимально возможную предельную надёжность, как и алгоритм $\boldsymbol{U}$ полного перебора решений. Однако средняя и максимальная трудоемкость $O(M)$ алгоритма $\boldsymbol{A}$ гораздо меньше, чем $O\left(M|B|^{n}\right)$ у алгоритма $\boldsymbol{U}$.

Интересные результаты справедливы для частного случая системы уравнений $S$ - случайной системы псевдобулевых уравнений со случайно модифицируемыми переменными (в псевдобулевых уравнениях переменные булевы, а значения функций из $\Phi_{i}, i=1, \ldots, m$, - действительные числа, т. е. $B=\{0,1\}, A \subset \boldsymbol{R}$ см. [3]). Случайная система псевдобулевых уравнений $S_{p}$ от- 
носительно $n$ неизвестных $x_{1}, \ldots, x_{n}$ состоит из $M$ выбираемых последовательно, случайно и независимо друг от друга уравнений вида

$$
f_{i, j}\left(x_{s_{1}} \oplus \delta_{s_{1}}, x_{s_{2}} \oplus \delta_{s_{2}}, \ldots, x_{s_{i}} \oplus \delta_{s_{i}}\right)=a .
$$

Вероятность появления уравнения с функцией $f_{i, j}$ в левой части и элементом $a$ в правой части равна $c_{i, j, a}$ для каждого набора $(i, j, a) \in E$. Каждый индекс $s_{1}, \ldots, s_{i}$ принимает значения $1, \ldots, n$ случайно и независимо от других индексов с вероятностями $p_{1}, \ldots, p_{n}$ соответственно, $i \in\{1, \ldots, m\}$. Все неизвестные $x_{s_{1}}, x_{s_{2}}, \ldots, x_{s_{i}}$ в каждом уравнении вида (5) модифицируются случайно, независимо от других уравнений и других переменных в этом уравнении, с помощью прибавления к ним аддитивных «добавков» $\delta_{s_{1}}, \delta_{s_{2}}, \ldots, \delta_{s_{i}}$ соответственно, являющихся независимыми, одинаково распределенными случайными величинами, принимающими значения 1 и 0 с вероятностями $р$ и $1-p$.

Возможны только два множества допустимых значений частично определяющихся из уравнений вида (5) переменных: $B_{1}=\{1\}$ или $B_{2}=\{0\}$. В соответствии с введенными выше обозначениями числа однозначно определяющихся из уравнения (3) переменных, равных 1 и 0 , равны $t_{i, j, a}\left(B_{1}\right)$, и $t_{i, j, a}\left(B_{2}\right)$ и

$$
\chi_{s}=\sum_{(i, j, a) \in E} c_{i, j, a} t_{i, j, a}\left(B_{s}\right), s=1,2 .
$$

Следствие 1. Случайная система уравнений $S_{p}$ имеет пороговую функциию совместности $\sqrt{n}$ тогда и только тогда, когда

$$
\chi(p)=p(1-p)\left(\chi_{1}-\chi_{2}\right)^{2}+\chi_{1} \chi_{2}>0 .
$$

Если $\chi(p)>0$, то при условии (4) справедливь оченки

$$
\mathbf{P}_{c}\left(S_{p}\right) \sim \exp \left\{-c^{2} \sigma^{2} \chi(p)\right\}, \pi_{n} \sim 1 .
$$

Условие $\chi(p)>0$ эквивалентно тому, что $\chi_{1}+\chi_{2}>0$ при $p(1-p)>0$ и $\chi_{1} \chi_{2}>0$ при $p(1-p)=0$.

Следствие 2. Почти все реализачии случайной системы уравнений $S_{p}$ совместны при $M=o\left(n^{2 / 3}\right)$ тогда и только тогда, когда $\chi(p)>0$.

Сформулированные результаты справедливы и в случае бесповторной выборки неизвестных, т. е. когда вероятность выбора неизвестных $x_{s_{1}}, x_{s_{2}}, \ldots, x_{s_{i}}$ для уравнения (3) определяется формулой

$$
p_{s_{1}} \frac{p_{s_{2}}}{\left(1-p_{s_{1}}\right)} \frac{p_{s_{3}}}{\left(1-p_{s_{1}}-p_{s_{2}}\right)} \ldots \frac{p_{s_{i}}}{\left(1-p_{s_{1}}-\ldots-p_{s_{i-1}}\right)} .
$$

Следствие 3. Утверждения теорем 1-3 и следствий 1, 2 справедливы при бесповторной выборке неизвестных. 
Выбор неизвестных для уравнения (3) осуществляется по схеме с возвращением. Если среди выбранных для него неизвестных нет совпадающих, то оно совместно. В противном случае некоторые уравнения могут, вообще говоря, оказаться несовместными. Так, например, булево уравнение $y_{1} \bar{y}_{2}=1$ несовместно при выборе для него двух одинаковых неизвестных. В доказательстве теоремы 1 будет показано, что при $M=o(n)$ почти все реализации случайной системы уравнений $S$ не имеют уравнений с совпадающими неизвестными и, следовательно, каждое такое уравнение совместно. Поэтому факт возможного выбора совпадающих неизвестных для уравнения (2) не оказывает влияния на получаемые в данной работе результаты.

Как и в [14], утверждения работы справедливы для случайных систем с известными в вариантах правыми частями уравнений, в частности, для случайных систем неравенств над конечным упорядоченным множеством, а также для случайных систем уравнений с векторными функциями. В первом случае множество $A$ значений функций в уравнениях случайной системы определяется как множество всех подмножеств некоторого конечного множества $D(|D| \geq 3)$, за исключением $D$ и $\varnothing$, а уравнения в формулах (3), (5) заменяются на соотношения $f_{i, j}\left(x_{s_{1}}, \ldots, x_{s_{i}}\right) \in a, f_{i, j}\left(x_{s_{1}} \oplus \delta_{s_{1}}, \ldots, x_{s_{i}} \oplus \delta_{s_{i}}\right) \in a$. Во втором случае $|D| \geq 2$ и множество $A$ определяются как объединение декартовых степеней множества $D: A=\bigcup_{i=1}^{t} D^{i}$.

Определение случайной системы уравнений $S$ содержит избыточные требования к ее вероятностной мере, которые не используются в доказательствах сформулированных результатов. Так, например, утверждение теоремы 2 справедливо, если выбор параметров $(i, j, a) \in E$ уравнения (3) осуществляется по любому вероятностному закону (вероятности $c_{i, j, a},(i, j, a) \in E$ могут быть неизвестны), но с вероятностью 1 каждое уравнение реализаций случайной системы уравнений $S$ не имеет частично определяющихся переменных. Подробнее этот вопрос рассмотрен в конце п. 4.

Г. В. Балакиным введены понятия порога несовместности и порога единственности - чисел уравнений, при которых система становится несовместной и среднее число решений не превосходит 1 (см. [3, с. 6]). В. А. Копытцевым введено понятие расстояния несовместности: число уравнений $M=M^{(\rho)}$, необходимое для определения несовместности с заданной (предельной) вероятностью $\rho, \rho>0$, случайно выбираемой реализации случайной системы уравнений $S$. При $\chi>0$ в соответствии с теоремой 1 расстояние несовместности имеет вид

$$
M^{(\rho)}=\sqrt{-\frac{n \ln (1-\rho)}{\sigma^{2} \chi}}+o(\sqrt{n}) .
$$


Оценка вероятности совместности случайной системы уравнений $S$ при равновероятной бесповторной выборке неизвестных в уравнениях получена ранее в теореме 4.1 статьи [14], случай $c_{m}=1, m=o(\sqrt{n})$ рассмотрен в $[1,4]$, случай двузначных неизвестных — в [16], алгоритм $\boldsymbol{A}-$ в [17]. Результаты и обзоры по тематике случайных систем уравнений можно найти в $[1-8,11-14,16]$. Понятие частично определяющихся переменных тесно связано с теорией полузапретов (см., например, $[10,14])$.

\section{3. Геометрические свойства случайной системы уравнений}

Для изучения геометрических свойств случайной системы уравнений каждой реализации случайной системы уравнений $S$ сопоставляется гиперграф (см. $[7,8,13,14])$. При этом переменной $x_{j}$ соответствует вершина $v_{j}$, $j=1, \ldots, n$, а каждое уравнение системы однозначно определяет соответствующее ему ребро сопоставляемого системе гиперграфа: ребро состоит из упорядоченных вершин, соответствующих существенным переменным функции в уравнении. Порядок на множестве вершин определяется порядком на множестве неизвестных соответствующего уравнения. Каждое ребро имеет метку, выбираемую из множества $E$. Метка ребра гиперграфа равна $(i, j, a)$ тогда и только тогда, когда в левой части соответствующего ему уравнения стоит функция $f_{i, j}$, а в правой части этого уравнения стоит элемент $a$. Установленное соответствие между системами уравнений и гиперграфами является взаимно однозначным. Случайной системе уравнений $S$ сопоставляется случайный гиперграф $M G(S)$, реализациями которого являются все гиперграфы, имеющие множество вершин $\left\{v_{1}, \ldots, v_{n}\right\}$ и $M$ упорядоченных ребер. Вершины в ребрах упорядочены, ребра имеют метки из множества $E$. Вероятности реализаций случайного гиперграфа $M G(S)$ равны вероятностям соответствующих им реализаций случайной системы уравнений $S$. Случайная система уравнений $S$ и соответствующий ей случайный гиперграф однозначно определяют друг друга.

Гиперграф $F$ называется подграфом гиперграфа $H$, если множества вершин и ребер $F$ являются подмножествами множеств вершин и ребер $H$. Изоморфный гиперграфу $F$ подграф гиперграфа $H$ называется $F$-графом. Ребро гиперграфа простое, если все его вершины различны, и особое в противном случае. Рангом ребра будем называть число инцидентных ему вершин, простым циклом называется цикл, образованный цепью без самопересечений (длины три или больше), в которой все ребра простые и каждая пара инцидентных ребер имеет ровно одну общую вершину. При автоморфизмах подграфов реализаций рассматриваемого случайного гиперграфа 2010, T. 1, № 3, С. 93-117 
предполагается сохранение номеров вершин в ребрах и инцидентность вершин и ребер. Будем говорить, что подсистема Н системы уравнений $F$ изолирована, если каждое уравнение подсистемы $H$ в системе уравнений $F$ не имеет общих неизвестных с уравнениями, не входящими в $H$.

Основной целью этого параграфа является доказательство того, что в условиях теоремы 1 почти все реализации случайной системы уравнений $S$ состоят только из изолированных уравнений и, может быть, некоторого числа пар уравнений, имеющих ровно одно общее неизвестное. В терминах теории случайных гиперграфов это утверждение означает отсутствие почти во всех реализациях случайного гиперграфа $M G(S)$ любых циклов и гипердеревьев из трех и более ребер. Любой связный гиперграф, имеющий цикл, содержит по крайней мере один из трех подграфов (см., например, [15]): особое ребро; пару ребер, пересекающихся по двум или более вершинам; простой цикл из трех ребер; простой цикл из четырех или более ребер (каждый такой цикл содержит гипердерево из трех ребер, являющееся простой цепью). В лемме 1 доказано, что все перечисленные подграфы (кроме гипердеревьев) отсутствуют почти во всех реализациях случайного гиперграфа не только при $M=o\left(n^{2 / 3}\right)$, но и при $M=o(n)$. В лемме 2 показано отсутствие почти во всех реализациях случайного гиперграфа гипердеревьев из трех и более ребер при $M=o\left(n^{2 / 3}\right)$. На эти характеристики случайного гиперграфа не влияют вид функций в левых частях уравнений и значения правых частей уравнений. Поэтому для упрощения формул будет использоваться эквивалентная форма определения случайной системы уравнений $S$. Для каждого уравнения сначала с вероятностями $c_{i}=\sum_{(j, a)} c_{i, j, a}, i=1, \ldots, m$ выбирается число $i$ неизвестных в функции уравнения. Затем с вероятностью $c_{i, j, a} / c_{i}$ осуществляется выбор номера $j$ функции из множества $\Phi_{i}$ для левой части уравнения и значения $a$ правой части для уравнения, зависящего от $i$ неизвестных. Процедура выбора неизвестных для уравнений приведена в исходном определении случайной системы уравнений $S$.

Лемма 1. При $M=o(n)$ почти все реализачии случайного гиперграфа $M G(S)$ не имеют особых ребер, пар ребер с двумя или более общими вершинами и простых ииклов из трех ребер.

Доказательство. Последовательно покажем отсутствие почти во всех реализациях случайного гиперграфа $M G(S)$ трех видов гиперграфов, приведенных в утверждении леммы.

Для каждого ребра случайного гиперграфа вероятность того, что оно является особым, равна $p^{*}=\sum_{i=2}^{m}\left(c_{i} \sum p_{s_{1}} \ldots p_{s_{i}}\right)$, где $c_{i}$ - вероятность того, что особое ребро имеет ранг $i, i=2, \ldots, m$, а суммирование во внутренней 
сумме ведется по всем упорядоченным наборам индексов $\left\langle s_{1}, \ldots, s_{i}\right\rangle$, выбираемым из множества $\{1, \ldots, n\}$ и имеющим не менее двух совпадающих индексов. Для получения среднего значения числа особых ребер $R^{*}$ в случайном гиперграфе нужно умножить эту вероятность на $M$. Справедлива оценка

$$
\mathbf{E} R^{*} \leq M \sum_{i=2}^{m}\left(c_{i} \sum_{r \neq t}\left(\sum_{s_{r}=1}^{n} p_{s_{r}}^{2} \sum_{\left\langle s_{1}, \ldots, s_{i}\right\rangle}\left(p_{s_{1}} \ldots p_{s_{i}} / p_{s_{r}} p_{s_{t}}\right)\right)\right) .
$$

Здесь во второй сумме индексы $r$ и $t$ - номера двух совпадающих вершин в ребре. Значение последней суммы в этой формуле не зависит от выбора номеров $r$ и $t$, а суммирование по оставшимся индексам можно разбить на независимые суммы, каждая из которых равна единице, поэтому при $M=o(n)$

$$
\mathbf{E} R^{*}=O\left(\frac{M}{n} \sum_{i=2}^{m}\left(\begin{array}{l}
i \\
2
\end{array}\right)\left(n \sum_{s_{r}=1}^{n} p_{s_{r}}^{2}\right)\right)=o(1) .
$$

Учитывая неравенство $\mathbf{P}\left(R^{*} \geq 1\right) \leq \mathbf{E} R^{*}$, убеждаемся, что в условиях леммы 1 почти все реализации случайного гиперграфа $M G(S)$ не имеют особых ребер.

С учетом этого результата при оценке числа пар ребер, имеющих две или более общих вершин, можно ограничиваться рассмотрением только пар простых ребер. Для среднего значения числа $R_{r, l}^{(s)}$ пар простых ребер ранга $r$ и $l$ в случайном гиперграфе, имеющих $s$ общих вершин, при $c_{r} c_{l}>0$, $s=2, \ldots, \min \{r, l\}$ справедлива оценка

$$
\mathbf{E} R_{r, l}^{(s)} \leq M(M-1) c_{r} c_{l} \sum p_{i_{1}} \ldots p_{i_{r}} p_{j_{1}} \ldots p_{j_{l}},
$$

в которой $c_{r} c_{l}-$ вероятность того, что любые два ребра случайного гиперграфа имеют ранги $r$ и $l$; множитель $M(M-1)$ - верхняя оценка числа различных вариантов пар номеров для этих двух ребер; суммирование осуществляется по всем наборам упорядоченных чисел $\left\langle i_{1}, \ldots, i_{r}\right\rangle,\left\langle j_{1}, \ldots, j_{l}\right\rangle$ из множества $\{1, \ldots, n\}$, имеющим ровно $s$ совпадающих элементов. Справедлива оценка

$$
\mathbf{E} R_{r, l}^{(s)} \leq M^{2} c_{r} c_{l} \sum \sum\left(\frac{p_{i_{1}} \ldots p_{i_{r}}}{p_{k_{1}} \ldots p_{k_{s}}} \frac{p_{j_{1}} \ldots p_{j_{l}}}{p_{t_{1}} \ldots p_{t_{s}}} p_{k_{1}}^{2} \ldots p_{k_{s}}^{2}\right),
$$

в первой сумме которой проводится суммирование по всем упорядоченным парам наборов чисел $\left\langle i_{1}, \ldots, i_{r}\right\rangle,\left\langle j_{1}, \ldots, j_{l}\right\rangle$ из множества $\{1, \ldots, n\}$, имеющим в пересечении ровно $s$ совпадающих элементов, а вторая сумма перебирает все наборы номеров $\left\langle k_{1}, \ldots, k_{s}\right\rangle,\left\langle t_{1}, \ldots, t_{s}\right\rangle$ мест в ребрах, соответствующих 
совпадающим элементам в наборах суммы: $k_{v}=t_{v}, v=1, \ldots, s$. Значения слагаемых внутренней суммы не изменяются при различном выборе номеров мест $\left\langle k_{1}, \ldots, k_{s}\right\rangle,\left\langle t_{1}, \ldots, t_{s}\right\rangle$ в ребрах, число таких слагаемых $(r)_{s}(l)_{s}$, а суммирование по оставшимся индексам можно разбить на независимые суммы, каждая из которых равна единице, поэтому

$$
\mathbf{E} R_{r, l}^{(s)} \leq \frac{M^{2} c_{r} c_{l}}{n^{s}}(r)_{s}(l)_{s} \prod_{v=1}^{s}\left(n \sum_{k_{v}=1}^{n} p_{k_{v}}^{2}\right)=o\left(n^{2-s}\right)=o(1), s=2, \ldots, \min \{r, l\} .
$$

Так как количество вариантов пар рангов ребер $r, l$ и числа $s$ совпадающих в них элементов конечно, то из этой формулы следует, что при $M=o(n)$ почти все реализации случайного гиперграфа $M G(S)$ не имеют пар ребер, имеющих две или более общих вершин.

Обозначим через $C(r, l, t)$ число простых циклов длины 3 в случайном гиперграфе $M G(S)$, образованных ребрами, имеющими ранги $r, l, t$. Для среднего значения этой случайной величины справедлива оценка

$$
\mathbf{E} C(r, l, t) \leq M(M-1)(M-2) c_{r} c_{l} c_{t} \sum p_{i_{1}} \ldots p_{i_{r}} p_{j_{1}} \ldots p_{j_{l}} p_{k_{1}} \ldots p_{k_{t}},
$$

в которой $c_{r} c_{l} c_{t}-$ вероятность того, что любые три ребра случайного гиперграфа имеют ранги $r, l$ и $t$; множитель $M(M-1)(M-2)-$ верхняя оценка числа различных вариантов номеров для этих трех ребер; суммирование осуществляется по всем упорядоченным тройкам наборов чисел $\left\langle i_{1}, \ldots, i_{r}\right\rangle$, $\left\langle j_{1}, \ldots, j_{l}\right\rangle,\left\langle k_{1}, \ldots, k_{t}\right\rangle$, пробегающих множество $\{1, \ldots, n\}$, причем каждая пара наборов имеет ровно один совпадающий элемент, а все три набора не имеют общих элементов.

В формуле (6) каждое слагаемое в сумме можно представить в виде

$$
\frac{p_{i_{1}} \ldots p_{i_{r}}}{p_{i_{1}^{*}} p_{i_{2}^{*}}^{*}} \frac{p_{j_{1}} \ldots p_{j_{l}}}{p_{j_{1}^{*}} p_{j_{2}^{*}}} \frac{p_{k_{1}} \ldots p_{k_{t}}}{p_{k_{1}^{*}} p_{k_{2}^{*}}} p_{i_{1}^{*}}^{2} p_{j_{1}^{*}}^{2} p_{k_{1}^{*}}^{2},
$$

где полагается, что $\left\{i_{1}^{*}, i_{2}^{*}\right\} \subseteq\left\{i_{1}, \ldots, i_{r}\right\},\left\{j_{1}^{*}, j_{2}^{*}\right\} \subseteq\left\{j_{1}, \ldots, j_{l}\right\},\left\{k_{1}^{*}, k_{2}^{*}\right\} \subseteq\left\{k_{1}, \ldots, k_{t}\right\}$, $i_{2}^{*}=j_{1}^{*}, j_{2}^{*}=k_{1}^{*}, k_{2}^{*}=i_{1}^{*}$. В (6) имеется $(r)_{2}(l)_{2}(t)_{2}$ вариантов выбора номеров совпадающих вершин в трех рассматриваемых ребрах. Сумму можно разбить на $r+l+t-3$ независимые суммы по индексам из множеств $\left\{i_{1}, \ldots, i_{r}\right\} \backslash\left\{i_{2}^{*}\right\},\left\{j_{1}, \ldots, j_{l}\right\} \backslash\left\{j_{2}^{*}\right\},\left\{k_{1}, \ldots, k_{t}\right\} \backslash\left\{k_{2}^{*}\right\}$. При этом суммы по каждому индексу $v$ из множеств $\left\{i_{1}, \ldots, i_{r}\right\} \backslash\left\{i_{1}^{*}, i_{2}^{*}\right\},\left\{j_{1}, \ldots, j_{l}\right\} \backslash\left\{j_{1}^{*}, j_{2}^{*}\right\},\left\{k_{1}, \ldots, k_{t}\right\} \backslash\left\{k_{1}^{*}, k_{2}^{*}\right\}$ имеют вид $\sum_{v=1}^{n} p_{v} \equiv 1$. Тогда справедлива оценка

$$
\mathbf{E} C(r, l, t) \leq \frac{M^{3}}{n^{3}} c_{r} c_{l} c_{t}(r)_{2}(l)_{2}(t)_{2}\left(n \sum_{i_{1}^{*}=1}^{n} p_{i_{1}^{*}}^{2}\right)\left(n \sum_{j_{1}^{*}=1}^{n} p_{j_{1}^{*}}^{2}\right)\left(n \sum_{k_{1}^{*}=1}^{n} p_{k_{1}^{*}}^{2}\right),
$$


из которой с учетом условия $M=o(n)$ и формулы (2) следует, что $\sum_{(r, l, t)} C(r, l, t)=o(1)$ и почти все реализации случайного гиперграфа $M G(S)$ не имеют простых циклов из трех ребер. Доказательство леммы 1 завершено.

Лемма 2. При $M=o\left(n^{2 / 3}\right)$ почти все реализачии случайного гиперграфа $M G(S)$ не имеют гипердеревьев из трех и более ребер.

Доказательство. Любое гипердерево из четырех и более ребер содержит подграф из трех ребер, являющийся гипердеревом, поэтому для доказательства леммы достаточно показать отсутствие почти во всех реализациях случайного гиперграфа $\mathrm{MG}(\mathrm{S})$ гипердеревьев из трех ребер. Гипердеревья из трех ребер могут иметь только вид трех ребер, пересекающихся по одной вершине, и простой цепи из трех ребер. Обозначим через $\mu_{3}(r, l, t)$ и $\tau_{3}(r, l, t)$ числа гипердеревьев из трех ребер с рангами $r, l, t$, имеющих одну общую вершину и являющихся простой цепью. Очевидно, что в случайном гиперграфе $\mathrm{MG}(\mathrm{S})$ число гипердеревьев из трех ребер равно $\sum_{r, l, t}\left(\mu_{3}(r, l, t)+\tau_{3}(r, l, t)\right)$. При каждом наборе рангов $r, l, t$ средние значения случайных величин $\mu_{3}(r, l, t)$ и $\tau_{3}(r, l, t)$ сверху можно оценить величиной

$$
M(M-1)(M-2) c_{r} c_{l} c_{t} \sum p_{i_{1}} \ldots p_{i_{r}} p_{j_{1}} \ldots p_{j_{l}} p_{k_{1}} \ldots p_{k_{t}},
$$

в которой суммирование осуществляется по всем тройкам наборов упорядоченных чисел $\left\langle i_{1}, \ldots, i_{r}\right\rangle,\left\langle j_{1}, \ldots, j_{l}\right\rangle,\left\langle k_{1}, \ldots, k_{t}\right\rangle$ из множества $\{1, \ldots, n\}$. В формуле (7) в каждом из этих трех наборов чисел отсутствуют повторения. Для упрощения выкладок такое требование к наборам чисел отбрасываем. При этом число слагаемых в сумме формулы (7) увеличивается. В случае $\mu_{3}(r, l, t)$ все три набора имеют ровно один элемент в их пересечении, причем любая пара наборов имеет общим только этот же элемент. В случае $\tau_{3}(r, l, t)$ первый и третий наборы не имеют общих элементов, а второй имеет с первым и третьим наборами ровно по одному элементу. Множитель $M(M-1)(M-2)$ является верхней оценкой числа различных вариантов пар номеров рассматриваемых ребер из всех ребер случайного гиперграфа, имеющих ранги $r, l, t$.

При оценке $\mathbf{E} \mu_{3}(r, l, t)$ каждое слагаемое суммы можно представить в виде

$$
\frac{p_{i_{1}} \ldots p_{i_{r}}}{p_{s}} \frac{p_{j_{1}} \ldots p_{j_{l}}}{p_{s}} \frac{p_{k_{1}} \ldots p_{k_{t}}}{p_{s}} p_{s}^{3},
$$

где полагается, что $s=\left\{i_{1}, \ldots, i_{r}\right\} \cap\left\{j_{1}, \ldots, j_{l}\right\} \cap\left\{k_{1}, \ldots, k_{t}\right\}$. В этом случае имеется rlt вариантов выбора номеров для общей вершины трех рассматриваемых ребер, а сумму можно разбить на $r+l+t-2$ независимых сумм по индексам из множеств $\left\{i_{1}, \ldots, i_{r}\right\},\left\{j_{1}, \ldots, j_{l}\right\} \backslash\{s\},\left\{k_{1}, \ldots, k_{t}\right\} \backslash\{s\}$. При этом сум2010, T. 1, № 3, C. 93-117 
мы по каждому индексу $v$, не равному $s$, имеют вид $\sum_{v=1}^{n} p_{v} \equiv 1$. Используя формулу (1), получаем

$$
\mathbf{E} \mu_{3}(r, l, t) \leq M^{3} c_{r} c_{l} c_{t} r l t \sum_{s=1}^{n} p_{s}^{3} \leq M^{3} n^{-2} r l t\left(n \sum_{s=1}^{n} p_{s}^{2}\right) \varepsilon_{2},
$$

откуда с учетом условия $M=o\left(n^{2 / 3}\right)$ и формулы (2) следует оценка $\mathbf{E} \mu_{3}(r, l, t)=o(1)$.

Перейдем к оценке среднего значения случайной величины $\mathbf{E} \tau_{3}(r, l, t)$. Каждое слагаемое в сумме формулы (7) можно представить в виде

$$
\frac{p_{i_{1}} \ldots p_{i_{r}}}{p_{j_{1}^{*}}} \frac{p_{j_{1}} \ldots p_{j_{l}}}{p_{j_{1}^{*}} p_{j_{2}^{*}}} \frac{p_{k_{1}} \ldots p_{k_{t}}}{p_{j_{2}^{*}}} p_{j_{1}^{*}}^{2} p_{j_{2}^{*}}^{2},
$$

где полагается, что $j_{1}^{*}=\left\{i_{1}, \ldots, i_{r}\right\} \cap\left\{j_{1}, \ldots, j_{l}\right\}, \quad j_{2}^{*}=\left\{j_{1}, \ldots, j_{l}\right\} \cap\left\{k_{1}, \ldots, k_{t}\right\}$. В этом случае в формуле (7) имеется $r l(l-1) t$ вариантов выбора номеров для общих вершин пар рассматриваемых ребер, а сумму можно разбить на $r+l+t-2$ независимых сумм по индексам из множеств $\left\{i_{1}, \ldots, i_{r}\right\}$, $\left\{j_{1}, \ldots, j_{l}\right\} \backslash\left\{j_{1}^{*}, j_{2}^{*}\right\},\left\{k_{1}, \ldots, k_{t}\right\}$. При этом суммы по каждому индексу $v$, не равному $j_{1}^{*}$ или $j_{2}^{*}$, имеют вид $\sum_{v=1}^{n} p_{v} \equiv 1$. Следовательно,

$$
\mathbf{E} \tau_{3}(r, l, t) \leq \frac{M^{3}}{n^{2}} c_{r} c_{l} c_{t} r l(l-1) t\left(n \sum_{j_{1}^{*}=1}^{n} p_{j_{1}^{*}}^{2}\right)\left(n \sum_{j_{2}^{*}=1}^{n} p_{j_{2}^{*}}^{2}\right),
$$

откуда с учетом условия $M=o\left(n^{2 / 3}\right)$ и формулы (2) следует оценка $\mathbf{E} \tau_{3}(r, l, t)=o(1)$.

Таким образом, $\mathbf{E}\left(\mu_{3}(r, l, t)+\tau_{3}(r, l, t)\right)=o(1)$, а параметры $r, l, t$ могут принимать конечное число различных вариантов. С учетом приведенного в начале доказательства замечания о гипердеревьях с тремя ребрами отсюда следует утверждение леммы 2.

Припишем $s$-й цвет $t_{i, j, a}\left(B_{s}\right)$ вершинам ребра, сопоставленного уравнению вида (3), которые соответствуют неизвестным в этом уравнении, имеющих множество допустимых значений $B_{s}, s=1, \ldots, h$. Наличие в некоторой реализации $H$ случайного гиперграфа $M G(S)$ гипердерева из двух ребер, имеющих общую вершину, которая в них окрашена в такие цвета $s$ и $k$, что $B_{s} \cap B_{k}=\varnothing$, является достаточным условием несовместности реализации случайной системы уравнений $S$, которая соответствует этому гиперграфу $H$. Этот факт позволяет получить оценки вероятности совместности случайной системы уравнений $S$. Для этого надо изучить предельное совместное распределение чисел $\xi_{s, k}=\xi_{s, k, n}$ вершин случайного гиперграфа $M G(S)$, которым в двух ребрах приписаны цвета $s, k, 1 \leq s \leq k \leq h$. Из определения случайной системы уравнений $S$ следует, что каждое ребро случайного ги- 
перграфа $M G(S)$ с вероятностью $c_{i, j, a}$ соответствует уравнению вида (3), состоит из $i$ вершин, из которых $t_{i, j, a}\left(B_{s}\right)$ приписан $s$-й цвет, $s=1, \ldots, h$. Изучение распределения случайных величин $\xi_{s, k}$ удобнее провести с использованием схемы размещения окрашенных частиц по $n$ ячейкам (см., например, [9]). Пусть имеется $N_{s}=N_{s}(n)$ частиц цвета $s, s=1, \ldots, h$. Каждая частица случайно и независимо от других частиц размещается с вероятностями $p_{1}, \ldots, p_{n}$ в ячейки с номерами $1, \ldots, n$. Обозначим через $\mu_{s, k}$ число ячеек в схеме размещения окрашенных частиц по ячейкам, которые содержат две частицы с $s$-м и $k$-м цветами, $1 \leq s \leq k \leq h$. При условии (4) ввиду отсутствия особых ребер почти во всех реализациях случайного гиперграфа $M G(S)$ предельное совместное распределение случайных величин $\xi_{s, k}, 1 \leq s \leq k \leq h$ такое же, как и предельное совместное распределение случайных величин $\mu_{s, k}$, $1 \leq s \leq k \leq h$, когда числа окрашенных частиц определяются формулами

$$
\begin{gathered}
N_{s} \sim c \sum_{(i, j, a) \in E} c_{i, j, a} t_{i, j, a}\left(B_{s}\right) \sqrt{n}=c \chi_{s} \sqrt{n} \text { при } \chi_{s}>0, s=1, \ldots, h, \\
N_{s}=0 \text { при } \chi_{s}=0, s=1, \ldots, h .
\end{gathered}
$$

Лемма 3. Совместные распределения случайных величин $\xi_{s, k}$, $1 \leq s \leq k \leq h$ и случайных величин $\mu_{s, k}, 1 \leq s \leq k \leq h$ при условии (4) сходятся каждое к многомерному распределению Пуассона с независимыми компонентами и средними значениями $\lambda_{s, k}=c^{2} \sigma^{2} \chi_{s} \chi_{k}$ при $1 \leq s<k \leq h$ и $\lambda_{s, s}=$ $=\left(c \sigma \chi_{s}\right)^{2} / 2,1 \leq s \leq h$.

Доказательство. Доказательство проведем методом моментов для случайных величин $\mu_{s, k}, 1 \leq s \leq k \leq h$. Обозначим через $I_{s, k}^{(j)}$ случайную величину, равную индикатору события, заключающегося в том, что в схеме размещения частиц ячейка с номером $j$ имеет ровно две частицы, причем с $s$-м и $k$-м цветами. Тогда $\mu_{s, k}=I_{s, k}^{(1)}+\ldots+I_{s, k}^{(n)}$ и

$$
\begin{gathered}
\mathbf{E} \mu_{s, k}=N_{s} N_{k} \sum_{j=1}^{n} p_{j}^{2}\left(1-p_{j}\right)^{M-2} \text { при } s \neq k, \\
\mathbf{E} \mu_{s, s}=\left(\begin{array}{c}
N_{s} \\
2
\end{array}\right) \sum_{j=1}^{n} p_{j}^{2}\left(1-p_{j}\right)^{M-2} .
\end{gathered}
$$

Здесь $p_{j}^{2}\left(1-p_{j}\right)^{M-2}$ - вероятность того, что в ячейку с номером $j$ попало ровно две частицы; $N_{s} N_{k}$ и $\left(\begin{array}{c}N_{s} \\ 2\end{array}\right)$ - числа вариантов номеров частиц с цветами $s$ и $k$, размещенных в эту ячейку при $s \neq k$ и $s=k$. Во втором случае частицы имеют одинаковые цвета, и перестановка пары сопоставляемых им номеров дает тот же самый вариант размещения этих частиц, поэтому число вариантов упорядоченных пар номеров $N_{s}\left(N_{s}-1\right)$ делится на 2. Учи- 
тывая формулы для $p_{j}$ и $M$, убеждаемся в справедливости оценок для средних значений исследуемых случайных величин

$$
\mathbf{E} \mu_{s, k}=c^{2} \chi_{s} \chi_{k}\left(n \sum_{j=1}^{n} p_{j}^{2}\right)(1+o(1))=c^{2} \sigma^{2} \chi_{s} \chi_{k}(1+o(1))=\lambda_{s, k}(1+o(1))
$$

при $s \neq k$,

$$
\mathbf{E} \mu_{s, s}=\frac{\left(c \chi_{s}\right)^{2}}{2}\left(n \sum_{j=1}^{n} p_{j}^{2}\right)(1+o(1))=\frac{\left(c \sigma \chi_{s}\right)^{2}}{2}(1+o(1))=\lambda_{s, s}(1+o(1)) .
$$

Зафиксируем набор $\mathbf{r}$ целых неотрицательных чисел $r_{s, k}, 1 \leq s \leq k \leq h$. Получим оценку смешанного факториального момента $\mathbf{E} \boldsymbol{\mu}_{\mathbf{r}}=\mathbf{E} \prod_{1 \leq s \leq k \leq h}\left(\mu_{s, k}\right)_{r_{s, k}}$ совместного распределения случайных величин $\mu_{s, k}, 1 \leq s \leq k \leq h$. Пусть

$$
\sum_{j=1}^{s-1} r_{j, s}+2 r_{s, s}+\sum_{k=s+1}^{h} r_{s, k}=r^{(s)}, s=1, \ldots, h .
$$

Тогда $r^{(1)}+\ldots+r^{(h)}=2 r$. Справедлива формула

$$
\mathbf{E} \boldsymbol{\mu}_{\mathbf{r}}=\sum_{\left\langle j_{1}, \ldots, j_{r}\right\rangle} \mathbf{E} I_{1,1}^{\left(j_{1}\right)} \ldots I_{1,1}^{\left(j_{\eta_{1,1}}\right)} I_{1,2}^{\left(j_{r_{1,1}+1}\right)} \ldots I_{1,2}^{\left(j_{r_{1,1}+\eta_{1,2}}\right)} \ldots I_{h, h}^{\left(j_{r-r_{h, h}+1}\right)} \ldots I_{h, h}^{\left(j_{r}\right)},
$$

в правой части которой ведется суммирование по всем выбираемым из множества $\{1, \ldots, n\}$ упорядоченным наборам индексов $\left\langle j_{1}, \ldots, j_{r}\right\rangle$, среди которых нет одинаковых. Первые $r_{1,1}$ элементов этих наборов соответствуют номерам ячеек, содержащих две частицы с цветом 1 , следующие $r_{1,2}$ элементов - номерам ячеек, содержащих частицы с цветами 1 и $2, \ldots$, последние $r_{h, h}$ элементов - номерам ячеек, содержащих две частицы с цветом $h$. Тогда

$$
\mathbf{E} \boldsymbol{\mu}_{\mathbf{r}}=\frac{\left(N_{1}\right)_{r^{(1)}} \ldots\left(N_{h}\right)_{r^{(h)}}}{2^{r_{1,1}+r_{2,2}+\ldots+r_{h, h}}} \sum_{\left\langle j_{1}, \ldots, j_{r}\right\rangle} p_{j_{1}}^{2} \ldots p_{j_{r}}^{2}\left(1-p_{j_{1}}-\ldots-p_{j_{r}}\right)^{M-2 r} .
$$

Здесь $p_{j_{1}}^{2} \ldots p_{j_{r}}^{2}\left(1-p_{j_{1}}-\ldots-p_{j_{r}}\right)^{M-2 r}-$ вероятность того, что все индикаторы $\left.I_{1,1}^{\left(j_{1}\right)}, \ldots, I_{1,2}^{\left(j_{\eta, 1}+1\right.}\right), \ldots, I_{h, h}^{\left(j_{r}\right)}$ равны единице, т. е. соответствующие ячейки содержат по две частицы с заданными цветами и не содержат других частиц; в дроби $\frac{\left(N_{1}\right)_{r^{(1)}} \ldots\left(N_{h}\right)_{r^{(h)}}}{2^{r_{1,1}+r_{2,2}+\ldots+r_{h, h}}}$ числитель равен числу вариантов упорядоченных наборов номеров частиц, для размещения пар частиц, $r^{(s)}$ из которых имеют цвет $s, s=1, \ldots, h$, а значение знаменателя обусловлено тем, что $r_{1,1}+\ldots+r_{h, h}$ рассматриваемых ячеек содержат пары неразличимых частиц с одинаковыми цветами, для которых выбираются неупорядоченные пары 
номеров. Учитывая формулы для $p_{j}, N_{j}, M$ и соотношение $r^{(1)}+\ldots+r^{(h)}=2 r$, убеждаемся, что

$$
\mathbf{E} \boldsymbol{\mu}_{\mathbf{r}}=\frac{\left(c \chi_{1}\right)^{r^{(1)}} \ldots\left(c \chi_{h}\right)^{r^{(h)}}}{2^{r_{1,1}+r_{2,2}+\ldots+r_{h, h}}} n^{r} \sum_{\left\langle j_{1}, \ldots, j_{r}\right\rangle} p_{j_{1}}^{2} \ldots p_{j_{r}}^{2}(1+o(1)) .
$$

В этой формуле сумму по упорядоченным наборам без повторений можно заменить на $r$ сумм по индексам $j_{1}, \ldots, j_{r}$, причем в первой сумме $j_{1}$ пробегает все значения $\{1, \ldots, n\}$; во второй сумме $j_{2}$ пробегает все эти значения, кроме $j_{1} ; \ldots ;$ в $r$-й сумме $j_{r}$ пробегает все эти значения, кроме $j_{1}, \ldots, j_{r-1}$ :

$$
\begin{gathered}
\mathbf{E} \boldsymbol{\mu}_{\mathbf{r}}=\frac{c^{2 r} \chi_{1}^{r^{(1)}} \ldots \chi_{h}^{r^{(h)}}}{2^{r_{1,1}+r_{2,2}+\ldots+r_{h, h}}}\left(n \sum_{j_{1}} p_{j_{1}}^{2}\left(n \sum_{j_{2} \neq j_{1}} p_{j_{2}}^{2} \ldots\left(n \sum_{j_{r} \notin\left\{j_{1}, \ldots, j_{r-1}\right\}} p_{j_{r}}^{2}\right) \ldots\right)\right)(1+o(1))= \\
=\frac{(c \sigma)^{2 r} \chi_{1}^{r^{(1)}} \ldots \chi_{h}^{r^{(h)}}}{2^{r_{1,1}+r_{2,2}+\ldots+r_{h, h}}}(1+o(1))=\prod_{1 \leq s<k \leq h}\left(c^{2} \sigma^{2} \chi_{s} \chi_{k}\right)^{r_{s, k}} \prod_{1 \leq s \leq h}\left(\frac{\left(c \sigma \chi_{s}\right)^{2}}{2}\right)^{r_{s, s}}(1+o(1)) .
\end{gathered}
$$

Следовательно, для всех наборов целых неотрицательных чисел $r_{s, k}$, $1 \leq s \leq k \leq h$ смешанные факториальные моменты $\mathbf{E} \boldsymbol{\mu}_{\mathrm{r}}$ совместного распределения случайных величин $\mu_{s, k}, 1 \leq s \leq k \leq h$ сходятся к соответствующим моментам многомерного распределения Пуассона с независимыми компонентами и приведенными в условии доказываемой леммы средними значениями. Утверждение леммы следует из теоремы 21 главы 1 книги [18].

\section{4. Доказательство основных результатов}

Доказательство теоремы 1. В замечании после следствия 3 отмечено, что выбор неизвестных для уравнений осуществляется по схеме с возвращением, поэтому некоторые уравнения могут, вообще говоря, оказаться несовместными. В лемме 1 показано, что при $M=o(n)$ почти все реализации случайного гиперграфа $M G(S)$ не имеют особых ребер. Следовательно, при $M=o(n)$ каждое отдельное уравнение почти всех реализаций случайной системы уравнений $S$ не имеет совпадающих неизвестных и совместно.

Из лемм 1, 2 следует, что при условии (4) почти все реализации случайного гиперграфа состоят из изолированных ребер и, возможно, некоторого количества пар уравнений, пересекающихся ровно по одной общей переменной. С учетом вышеизложенного, в этих условиях несовместность почти всех реализаций случайной системы уравнений может быть обусловлена только подсистемами, состоящими из двух уравнений, пересекающихся ровно по одной общей переменной. Такие подсистемы противоречивы тогда и только тогда, когда общие переменные их уравнений частично определяются в каждом из этих уравнений и множества допустимых значений этих переменных, определяемых из первого и второго уравнений, не имеют 
общих элементов, т.е. при $\chi>0$, когда найдутся такие $\left(i_{1}, j_{1}, a_{1}\right),\left(i_{2}, j_{2}, a_{2}\right), s$, $k$, что $c_{i_{1}, j_{1}, a_{1}} t_{i_{1}, j_{1}, a_{1}}\left(B_{s}\right) c_{i_{1}, j_{1}, a_{1}} t_{i_{2}, j_{2}, a_{2}}\left(B_{k}\right)>0, B_{s} \cap B_{k}=\varnothing$. Предельное распределение чисел таких переменных определяется результатом леммы 3. Следовательно, справедлива оценка

$$
\mathbf{P}_{c}(S)=\mathbf{P}\left(\sum_{B_{s} \cap B_{k}=\varnothing} \xi_{s, k}=0\right)+o(1)=\exp \left\{-\sum_{B_{s} \cap B_{k}=\varnothing} \lambda_{s, k}\right\}+o(1) .
$$

С учетом формул для $\lambda_{s, k}$, приведенных в утверждении леммы 3 , отсюда следует искомая оценка вероятности совместности случайной системы уравнений $S$ при условии $\chi>0$. Эта оценка верна и при $\chi=0$, так как в этом случае почти все реализации случайной системы уравнений $S$ совместны.

Перейдем к обоснованию условия наличия пороговой функции совместности случайной системы уравнений $S$. Из лемм 1 и 2 следует, что при $M=o(\sqrt{n})$ почти все реализации случайного гиперграфа состоят из изолированных ребер. Тогда при $M=o(\sqrt{n})$ почти все реализации случайной системы уравнений $S$ совместны при любых $\chi$, так как состоят из изолированных уравнений, каждое из которых совместно почти во всех реализациях случайной системы уравнений $S$. Если же $M / \sqrt{n} \rightarrow \infty$ и $\chi>0$, то почти все реализации случайной системы уравнений $S$ несовместны, так как из леммы 3 следует, что в этих условиях почти все реализации случайного гиперграфа содержат вершины, которым приписаны такие цвета $s$ и $k$, что $B_{s} \cap B_{k}=\varnothing$. Следовательно, условие $\chi>0$ является достаточным для того, чтобы случайная система уравнений $S$ имела пороговую функцию совместности $\sqrt{n}$. В соответствии с леммами 1 и 2 при $M / \sqrt{n} \rightarrow \infty, M=o\left(n^{2 / 3}\right)$ почти все реализации случайной системы уравнений $S$ состоят только из изолированных уравнений и изолированных подсистем из двух уравнений, пересекающихся ровно по одной вершине. Выше было показано, что изолированные уравнения совместны почти во всех реализациях случайной системы уравнений $S$, а при условии $\chi=0$ совместны и все изолированные подсистемы из двух уравнений, пересекающихся ровно по одной вершине. Таким образом, условие $\chi>0$ необходимо и достаточно для наличия пороговой функции совместности вида $\sqrt{n}$ у случайной системы уравнений $S$. Доказательство теоремы 1 завершено.

Доказательство теоремы 2. Из лемм 1,2 следует, что при $M=o\left(n^{2 / 3}\right)$ почти все реализации случайной системы уравнений $S$ состоят из изолированных подсистем из одного уравнения или из двух уравнений, имеющих ровно по одной общей переменной. В доказательстве теоремы 1 было показано, что при условии $\chi=0$ совместны все изолированные подсистемы 
из двух уравнений, пересекающихся ровно по одному неизвестному, а изолированные уравнения совместны почти во всех реализациях случайной системы уравнений $S$ при $M=o(n)$. Отсюда следует утверждение теоремы 2.

Доказательство теоремы 3. Алгоритм $\boldsymbol{A}$ осуществляет поиск двух несовместных ограничений на одно неизвестное системы уравнений, предельные распределения которых описаны в лемме 3. Поэтому в условиях теоремы 3 справедлива оценка

$$
\pi_{n}=\mathbf{P}\left(\sum_{B_{s} \cap B_{k}=\varnothing} \xi_{s, k}>0\right) /\left(1-\mathbf{P}_{c}(S)\right) .
$$

В условиях теоремы 3 из теоремы 1 и леммы 2 следует формула

$$
\mathbf{P}\left(\sum_{B_{s} \cap B_{k}=\varnothing} \xi_{s, k}>0\right) \sim\left(1-\mathbf{P}_{c}(S)\right) .
$$

Из двух последних формул следует оценка для $\pi_{n}$. Вторая часть утверждения теоремы 3 следует из полученных в доказательстве теоремы 1 фактов: при $M=o\left(n^{2 / 3}\right)$ почти все реализации случайной системы уравнений $S$ состоят из изолированных подсистем из одного уравнения или из двух уравнений, имеющих ровно одну общую переменную; отдельные уравнения почти всех реализаций случайной системы уравнений $S$ совместны; приведенное в утверждении теоремы 3 описание несовместных изолированных подсистем из двух уравнений с одной общей переменной получено в доказательстве теоремы 1.

Доказательство следствия 1. Для случайной системы уравнений $S_{p}$ только два множества $B_{1}=\{1\}$ и $B_{2}=\{0\}$ определяют ограничения на значения неизвестных. В случае $p=0$ из теорем 1,3 следует утверждение следствия 1 , причем $\chi(p)=c^{2} \chi_{1} \chi_{2}$, где $\chi_{1}$ и $\chi_{2}$ определены перед формулировкой следствия. Случай $p=1$ симметричен случаю $p=0$, при этом меняются местами множества $B_{1}, B_{2}$ и величины $\chi_{1}, \chi_{2}$, а все утверждения остаются такими же.

Получим утверждение следствия 1 при $p(1-p)>0$, используя имеющийся результат при $p=0$. Введем случайные величины $\alpha_{0}=\alpha_{0, n}=\xi_{1,1}+\xi_{2,2}$ и $\alpha_{1}=\alpha_{1, n}=\xi_{1,2}$, равные числу неизвестных в реализациях случайной системы уравнений $S_{p}$, на значения которых наложены два совместных и два несовместных ограничения при $p=0$. Из леммы 3 следует, что распределение векторной случайной величины $\left(\alpha_{0}, \alpha_{1}\right)$ слабо сходится к двумерному распределению Пуассона с независимыми компонентами и параметрами $\left(\lambda_{1,1}+\lambda_{2,2}, \lambda_{1,2}\right)$. При переходе от случая $p=0$ к случаю $0<p<1$ из-за аддитивных «добавков» $\delta_{s_{1}}, \delta_{s_{2}}, \ldots, \delta_{s_{i}}$ в уравнении (5) каждое определившееся значение 0 или 1 неизвестного с вероятностью $1-p$ не изменяется и с вероятностью $p$ меняется. При этом каждая пара определившихся значений с вероятностью $p^{2}+(1-p)^{2}$ не меняет своей совместности или несовместности 
и меняет ее с вероятностью $2 p(1-p)$. Тогда случайную величину $\alpha=\alpha_{n}$, равную числу неизвестных случайной системы уравнений $S_{p}$ при $0<p<1$, которые при этом будут иметь пару несовместных значений 0 и 1 , можно определить как

$$
\alpha=\sum_{i=1}^{\alpha_{0}} \psi_{0, i}+\sum_{j=1}^{\alpha_{1}}\left(1-\psi_{1, j}\right),
$$

где $\psi_{0,1}, \psi_{0,2}, \ldots, \psi_{1,1}, \psi_{1,2}, \ldots$ - независимые в совокупности и не зависящие от $\alpha_{0}, \alpha_{1}$ случайные величины, имеющие одинаковое распределение и принимающие значения 1 и 0 с вероятностями $2 p(1-p)$ и $p^{2}+(1-p)^{2}$ соответственно. Из леммы 3 и определения случайных величин $\psi_{0,1}, \psi_{0,2}, \ldots, \psi_{1,1}, \psi_{1,2}, \ldots$ следует, что распределение случайной величины $\alpha$ слабо сходится к распределению Пуассона со средним значением $\left(p^{2}+(1-p)^{2}\right) \lambda_{1,2}+2 p(1-p)\left(\lambda_{1,1}+\lambda_{2,2}\right)$. Подставив в это выражение оценки $\lambda_{1,2}=c^{2} \sigma^{2} \chi_{1} \chi_{2}, \lambda_{s, s}=\left(c \sigma \chi_{s}\right)^{2} / 2, s=1,2$, получаем формулу $\mathbf{E} \alpha=c^{2} \sigma^{2}\left(\chi_{1} \chi_{2}+p(1-p)\left(\chi_{1}-\chi_{2}\right)^{2}\right)$. Отсюда и из формулы $\mathbf{P}_{c}\left(S_{p}\right)=\mathbf{P}_{c}(\alpha=0)+o(1)$ следует оценка для вероятности совместности случайной системы уравнений $S$ при $p(1-p)>0$. Оценка для надежности алгоритма $\boldsymbol{A}$ следует из оценки для вероятности совместности случайной системы уравнений $S$ и теоремы 3 .

Доказательство следствия 2. Утверждение следствия 2 следует из теоремы 2 , следствия 1 и из того, что случайная система уравнений $S_{p}$ является частным случаем случайной системы уравнений $S$.

Доказательство следствия 3. Утверждение следствия 3 следует из леммы 1 , в которой показано отсутствие особых ребер почти во всех реализациях случайного гиперграфа $M G(S)$ при $M=o(n)$. Для случайной системы уравнений $S$ это означает отсутствие почти во всех ее реализациях уравнений с неоднократным вхождением неизвестных.

В формулировках и доказательствах основных результатов используется только часть информации о вероятностной мере на множестве реализаций случайной системы уравнений $S$. Так, например, вероятности $c_{i, j, a}$ для наборов $(i, j, a) \in E$, которым соответствуют уравнения вида (3) без частично определяющихся переменных, не используются. Доказанные результаты справедливы для определяемой ниже случайной системы уравнений $S^{*}$ и для системы $S_{p}^{*}$, получаемой аналогично из $S_{p}$.

Пусть определены вероятности $c_{i}, i=1, \ldots, m$, сумма которых равна единице. Вероятности $c_{i, j, a}$ определены только для таких наборов $(i, j, a) \in E$, для которых найдется набор $\left(i_{1}, j_{1}, a_{1}\right) \in E$ и множества $B_{s}, B_{k}$ такие, что 
$B_{s} \cap B_{k}=\varnothing$ и $t_{i, j, a}\left(B_{s}\right) t_{i_{1}, j_{1}, a_{1}}\left(B_{k}\right)>0$. Допускается случай $(i, j, a)=\left(i_{1}, j_{1}, a_{1}\right)$. Если $j>m_{i}$ или $a$ не принадлежит множеству значений функции $f_{i, j}$, то $c_{i, j, a}=0$. Определим множества $E_{i}=\{i\} \times\left\{1,2, \ldots, m_{i}\right\} \times A, i=1, \ldots, m$. Пусть $E_{i}^{*}-$ множество таких наборов $(i, j, a) \in E_{i}$, для которых определены вероятности $c_{i, j, a}$, и $c_{i}^{*}=\sum_{(i, j, a) \in E_{i}^{*}} c_{i, j, a}$.

Уравнения в случайной системе уравнений $S^{*}$ выбираются последовательно, вероятность выбора уравнения, зависящего от $i$ неизвестных, равна $c_{i}, i=1, \ldots, m$. При условии, что выбираемое уравнение зависит от $i$ неизвестных, с вероятностью $c_{i, j, a} / c_{i}$ уравнение имеет вид (3) для всех $(i, j, a) \in E_{i}^{*}$, с вероятностью $\left(1-c_{i}^{*}\right) / c_{i}$ уравнение имеет вид (3), где $(i, j, a) \in E_{i} \backslash E_{i}^{*}$, а процедура выбора конкретного набора $(i, j, a)$ может быть любой, в том числе - зависящей от других уравнений и детерминированной. Выбор индексов неизвестных для уравнений осуществляется так же, как и в случайной системе уравнений $S$.

Теоремы 1-3 справедливы для случайной системы уравнений $S^{*}$, а следствия 1-3 - для случайной системы уравнений $S_{p}^{*}$, так как в леммах 1,2 требуются только вероятности $c_{1}, \ldots, c_{m}$ и $p_{1}, \ldots, p_{n}$. Для доказательства утверждений теорем 1-3 в утверждении леммы 3 используются только те случайные величины $\mu_{s, k}$, для которых $B_{s} \cap B_{k}=\varnothing$ и $\chi_{s} \chi_{k}>0$. Для определения таких $\chi_{s}, \chi_{k}$ достаточно определить вероятности $c_{i, j, a}$ только для таких пар наборов $(i, j, a),\left(i_{1}, j_{1}, a_{1}\right)$, для которых $t_{i, j, a}\left(B_{s}\right) t_{i_{1}, j_{1}, a_{1}}\left(B_{k}\right)>0$.

\section{5. Примеры}

ПРимЕР 1. Случайная система $S$ состоит из уравнений вида

$$
f_{i, 1}\left(x_{s_{1}}, \ldots, x_{s_{i}}\right)=x_{s_{1}}+\ldots+x_{s_{i}}=a,
$$

где $i \in\{1,2, \ldots, m\}$ (т. е. $c_{i, j, a}=0$ при $j>1$ ), причем неизвестные принимают значения в множестве $\{0,1, \ldots, q-1\}$, операция сложения определена в множестве действительных чисел, а правая часть такого уравнения может принимать значения только из множества $\{0,1, \ldots,(q-1) i\}$. Тогда справедливы утверждения теорем $1-3$, причем

$$
\chi=c^{2} \sum_{(i, j) ; 0 \leq s_{1}<s_{2} \leq q-1} i j c_{i, 1, s_{1}} c_{j, 1,(q-1)(j-1)+s_{2}} .
$$


ПРИМЕР 2. Случайная система $S_{p}$ состоит из булевых уравнений вида

$$
f_{i, s}\left(y_{1}, \ldots, y_{i}\right)=y_{1} \ldots y_{s} \bar{y}_{s+1} \ldots \bar{y}_{i}=a, a \in\{0,1\}, 1 \leq i \leq m, 0 \leq s \leq i,
$$

т. е. $c_{i, j, a}=0$ при $j>i$. Тогда справедливы утверждения следствий 1 и 2 , где

$$
\chi_{1}=c_{1,1,0}+\sum_{i=1}^{m} \sum_{s=0}^{i-1} c_{i, s, 1}(i-s), \chi_{2}=c_{1,0,0}+\sum_{i=1}^{m} \sum_{s=1}^{i} c_{i, s, 1} s .
$$

Условие $\chi(p)>0$ выполняется, если, например, найдется пара $(i, s)$, для которой $c_{i, s, 1}>0,0<s<i$. вида

ПРИМЕР 3. Случайная система $S_{p}$ состоит из псевдобулевых уравнений

$$
f_{s d, 1}\left(y_{1}, \ldots, y_{s d}\right)=\sum_{k=0}^{s-1} y_{k d+1} \vee \ldots \vee y_{k d+l} \vee \bar{y}_{k d+l+1} \vee \ldots \vee \bar{y}_{k d+d}=a,
$$

$d \geq 1, \quad 1 \leq s \leq t=\lfloor m / d\rfloor, \quad 1 \leq l<d ; \quad$ т. е. $\quad c_{i, j, a}=0 \quad$ при $\quad i \neq s d, \quad s \leq t=\lfloor m / d\rfloor$ и при $j>1$. Здесь $f_{s d, 1}\left(y_{1}, \ldots, y_{s k}\right)-$ псевдобулева функция, равная сумме $s$ булевых функций в поле действительных чисел; правая часть $a$ уравнения вида (8) принимает значения в множестве $\{0,1, \ldots, s\}$. Справедливы утверждения следствий 1,2 , где

$$
\chi_{1}=l \sum_{s=1}^{t} s c_{s d, 1,0}, \quad \chi_{2}=(d-l) \sum_{s=1}^{t} s c_{s d, 1,0} .
$$

Условие $\chi(p)>0$ выполняется тогда и только тогда, когда найдется такое $s, 1 \leq s \leq t=\lfloor m / d\rfloor$, что $c_{s d, 1,0}>0$.

ПРИМЕР 4. Функции в уравнениях случайной системы $S$ над конечным полем имеют вид элементарных симметрических многочленов

$$
\sum_{1 \leq i_{1}<i_{2}<\ldots<i_{k} \leq m} y_{i_{1}} y_{i_{2}} \ldots y_{i_{k}},
$$

где $1 \leq k \leq m-1$. В этом случае при $M=o\left(n^{2 / 3}\right)$ почти все реализации случайной системы уравнений $S$ совместны.

ПРИМЕР 5. Функции в уравнениях случайной системы $S$ имеют вид $f_{i, 1}\left(y_{1}, \ldots, y_{i}\right)=\max \left\{y_{1}, \ldots, y_{i}\right\}$ или $f_{i, 2}\left(y_{1}, \ldots, y_{i}\right)=\min \left\{y_{1}, \ldots, y_{i}\right\}, i=2, \ldots, m$; множества значений аргументов и функций одинаковы, на них задано отношение порядка: $b_{1}<b_{2}<\ldots<b_{|B|}$. Тогда справедливы утверждения теорем $1-3$, причем

$$
\chi=\sum_{i=2}^{m} \sum_{j=2}^{m} \sum_{b_{1} \leq a_{1}<a_{2} \leq b_{|B|}} c_{i, a_{1}} c_{j, 2, a_{2}} .
$$

Условие $\chi>0$ выполняется тогда и только тогда, когда найдутся наборы $\left(i, a_{1}\right),\left(j, a_{2}\right)$, для которых $a_{1}<a_{2}, c_{i, 1, a_{1}}>0, c_{j, 2, a_{2}}>0$. 
ПРИМЕР 6. Случайная система уравнений $S$ рассматривается над кольцом вычетов $\mathrm{Z} / 4=\{0,1,2,3\}$, функции в уравнениях имеют вид $f_{2,1}\left(y_{1}, y_{2}\right)=y_{1}\left(y_{2}+1\right)$. Тогда $\chi=\left(c_{2,1,1}+c_{2,1,3}\right)^{2}$ и справедливы теоремы $1-3$.

ПримЕР 7. Случайная система уравнений $S$ рассматривается над кольцом вычетов $Z / 4$, функции в ее уравнениях имеют вид $f_{2,2}\left(y_{1}, y_{2}\right)=y_{1}^{2}+y_{2}^{2}$, $B=\{0,1,2\}$. Тогда $\chi=4 c_{2,2,0} c_{2,2,2}$ и справедливы утверждения теорем $1-3$.

ПримЕР 8. Случайная система уравнений $S$ рассматривается над кольцом вычетов $\mathrm{Z} / q, q-$ четно, $q \geq 4$, функции в ее уравнениях имеют вид $f_{2,3}\left(y_{1}, y_{2}\right)=y_{1}-2 y_{2}$. Тогда справедливы утверждения теорем $1-3$, причем

$$
\chi=\sum_{i=0}^{q / 2-1} c_{2,2,2 i} \sum_{j=0}^{q / 2-1} c_{2,2,2 j+1} .
$$

Приведенная в примере 1 оценка параметра $\chi$ получена в примере 4.1 статьи [14] при исследовании близкой случайной системы уравнений, но с равновероятной бесповторной выборкой неизвестных. Там же в примеpax 4.2, 4.3 получены оценки параметров $\chi_{1}, \chi_{2}$ для случайных систем с приведенными в примерах 2, 3 уравнениями при условии $p=0$ и при равновероятной бесповторной выборке неизвестных. Выкладки при оценке параметров $\chi_{1}, \chi_{2}$ остаются без изменений и в данной работе не повторяются (для $\chi_{1}$ и $\chi_{2}$ в [14] использованы обозначения $c^{(1)}$ и $c^{(2)}$ соответственно). В примере 4 уравнения не имеют частично определяющихся переменных, поэтому $\chi=0$ и выполняется теорема 2 . В примере 5 все переменные уравнения $\max \left\{x_{s_{1}}, \ldots, x_{s_{i}}\right\}=b_{k}$ при $k<|B|$ частично определяются и имеют множество допустимых значений $\left\{b_{1}, b_{2}, \ldots, b_{k}\right\}$. Аналогично, все переменные уравнения $\min \left\{x_{s_{1}}, \ldots, x_{s_{i}}\right\}=b_{t}$ при $t>1$ имеют множество допустимых значений $\left\{b_{t}, b_{t+1}, \ldots, b_{|B|}\right\}$. В такой системе указанные пары допустимых значений несовместны при $b_{k}<b_{t}$, других несовместных пар допустимых значений нет. Тогда $\chi$ имеет приведенный вид и справедливы теоремы 1-3.

Таблицы значений функций $f_{2,1}$ и $f_{2,2}$ в примерах 6 и 7 имеют следующий вид

\begin{tabular}{|c|c|c|c|c|}
\hline$y_{1} y_{2}$ & 0 & 1 & 2 & 3 \\
\hline 0 & 0 & 0 & 0 & 0 \\
\hline 1 & 1 & 2 & 3 & 0 \\
\hline 2 & 2 & 0 & 2 & 0 \\
\hline 3 & 3 & 2 & 1 & 0 \\
\hline
\end{tabular}

Таблица значений функции $f_{2,1}$.

\begin{tabular}{|c|c|c|c|c|}
\hline$y_{1} y_{2}$ & 0 & 1 & 2 & 3 \\
\hline 0 & 0 & 1 & 0 & 1 \\
\hline 1 & 1 & 2 & 1 & 2 \\
\hline 2 & 0 & 1 & 0 & 1 \\
\hline 3 & 1 & 2 & 1 & 2 \\
\hline
\end{tabular}

Таблица значений функции $f_{2,2}$. 
В примере 6 уравнение (3) с 0 в правой части не имеет частично определяющихся переменных. Если правая часть уравнения (3) с функцией $f_{2,1}$ равна 1,2 и 3 , то первая переменная имеет множества допустимых значений $B_{1}=\{1,3\},\{1,2,3\}$ и $B_{1}$, а вторая переменная $-B_{2}=\{0,2\},\{0,1,2\}$ и $B_{2}$. Только $B_{1}$ и $B_{2}$ имеют пустое пересечение. Так как $t_{2,1,1}\left(B_{1}\right)=t_{2,1,3}\left(B_{1}\right)=1$ и $t_{2,1,1}\left(B_{2}\right)=t_{2,1,3}\left(B_{2}\right)=1, \quad$ то $\quad \chi_{1}=\chi_{2}=c_{2,1,1}+c_{2,1,3} \quad$ и $\quad \chi=\chi_{1} \chi_{2}=\left(c_{2,1,1}+c_{2,1,3}\right)^{2}$. $\mathrm{B}$ примере 7 уравнение (3) с 1 в правой части не имеет частично определяющихся переменных. Если правая часть уравнения (3) с функцией $f_{2,2}$ равна 2 и 0 , то первая и вторая переменные уравнения имеют непересекающиеся множества допустимых значений $B_{1}=\{1,3\}$ и $B_{2}=\{0,2\}$. Так как $t_{2,2,2}\left(B_{1}\right)=t_{2,2,0}\left(B_{2}\right)=2$, то $\chi_{1}=2 c_{2,2,2}, \chi_{2}=2 c_{2,2,0}$ и $\chi=\chi_{1} \chi_{2}=4 c_{2,2,0} c_{2,2,2}$.

В примере 8 уравнение (3) эквивалентно уравнению $x_{s_{1}}=a+2 x_{s_{2}}$. Вторая переменная в этом уравнении не имеет запретных значений, а первая переменная ввиду четности модуля $q$ кольца вычетов частично определяется: при четных $a$ множество ее допустимых значений есть $B_{3}=\{0,2, \ldots$, $2 q-2\}$, а при нечетных $a-B_{4}=B \backslash B_{3}=\{1,3, \ldots, 2 q-1\}$. Так как $t_{2,2, a}\left(B_{3}\right)=1$ при четных $a$ и $t_{2,2, a}\left(B_{4}\right)=1$ при нечетных $a$, то $\chi=\chi_{3} \chi_{4}$, где

$$
\chi_{3}=\sum_{i=0}^{q / 2-1} c_{2,2,2 i}, \chi_{4}=\sum_{j=0}^{q / 2-1} c_{2,2,2 j+1} .
$$

При равновероятной бесповторной выборке неизвестных оценки вероятности совместности случайной системы $S$ получены $Г$. В. Балакиным в случае уравнений вида $y_{1}+\ldots+y_{s}=a$ с операцией сложения в поле действительных чисел и $B=\{1, \ldots, q\}$, булевых уравнений вида $y_{1} \vee y_{2}$, $y_{1} \vee \bar{y}_{2}$ (см. [2]), В. А. Копытцевым - в случаях псевдобулевых уравнений вида $y_{1} \vee y_{2}+\ldots+y_{2 s-1} \vee y_{2 s}, \quad y_{1} \vee \bar{y}_{2}+\ldots+y_{2 s-1} \vee \bar{y}_{2 s}$, случаи булевых уравнений вида $\quad y_{1} \ldots y_{s} \bar{y}_{s+1} \ldots \bar{y}_{m}, \quad y_{1} \ldots y_{m}, \quad y_{1}\left(y_{2} \oplus \ldots \oplus y_{m}\right), \quad \bar{y}_{1}\left(y_{2} \oplus \ldots \oplus y_{m}\right)$, $\left(y_{1} \oplus y_{2}\right)\left(y_{3} \oplus \ldots \oplus y_{m}\right)$ и ряд других рассмотрены в работах $[13,14,16]$, случайные системы линейных уравнений исследовались, в частности, в [1$8,11-13]$.

\section{Список литературы}

1. Balakin G. $V$. On the number of solutions of systems of pseudo-boolean random equations. - В сб.: Вероятностные методы дискретной математики. M.-Utrecht: ТВП-VSP, 1993, c. 71-98.

2. Балакин Г. В. Графы систем двучленных уравнений с булевыми неизвестными. - Теория вероятн. примен., 1995, т. 40, в. 2, с. 241-259.

3. Балакин Г. В. Введение в теорию случайных систем уравнений. - В сб.: Труды по дискретной математике. Т. 1. - М.: ТВП, 1997, с. 1-18. 
4. Балакин Г. В. Системы случайных уравнений над конечным полем. В сб.: Труды по дискретной математике. Т. 2. - М.: ТВП, 1998, с. 21-37.

5. Балакин Г. В., Колчин В. Ф., Хохлов В. И. Гиперциклы в случайном гиперграфе. - Дискретн. матем., 1991, т. 3, в. 3, с. 102-108.

6. Коваленко И. Н., Левитская А. А., Савчук М. Н. Избранные задачи вероятностной комбинаторики. - Киев: Наукова думка, 1986, с. 224.

7. Колчин В. Ф. Системы случайных уравнений. - М.: МИЭМ, 1988.

8. Колчин В. Ф. Случайные графы. - М.: ФИЗМАТЛИТ, 2000.

9. Колчин В. Ф., Севастьянов Б. А., Чистяков В. П. Случайные размещения. - М.: Наука, 1976, с. 224.

10. Никонов В. Г., Никонов Н. В. Запреты $k$-значных функций и их связь с проблемой разрешимости систем уравнений специального вида. Вестник РУДН, сер. Прикл. матем. и компьют. матем., 2003, т. 2, в. 1, c. 79-93.

11. Сачков В. Н. Случайные покрытия и системы функциональных уравнений. - Интеллектуальные системы, 1997, т. 2, в. 1-4, с. 297-315.

12. Сачков B. Н. Случайные неравновероятные покрытия и функциональные уравнения. - В сб.: Труды по дискретной математике. Т. 5. - М.: ФИЗМАТЛИТ, 2002, с. 205-218.

13. Шаповалов А. В. Вероятность совместности случайных систем булевых уравнений. - Дискрет. матем., 1995, т. 7, в. 3, с. 146-159.

14. Шаповалов А. В. Пороговые функции совместности случайных систем уравнений. - В сб.: Труды по дискретной математике. Т. 9. М.: Гелиос APB, 2006, с. 377-400.

15. Шаповалов A. В. Цикловая структура случайного неоднородного гиперграфа на докритическом этапе эволюции. - Дискретн. матем., 2007, т. 19 , в. 4, с. $52-69$.

16. Шаповалов A. В. Совместность и алгоритм распознавания несовместности реализаций случайных систем дискретных уравнений с двузначными неизвестными. - Дискретн. матем., 2008, т. 20, в. 3, с. 28-39.

17. Шаповалов A. В. Совместность и алгоритм распознавания несовместности реализаций случайной системы дискретных уравнений с неравновероятной выборкой неизвестных. - Обозрение прикладной и промышленной математики, 2009, т. 16, в. 2, с. 280-281.

18. Bollobas B. Random graphs. — London etc.: Academic Press, 1985. 

